Artigo Original

Original Article

Fernanda Maria Santana Bomfim ${ }^{1}$ Brasília Maria Chiari² Francelise Pivetta Roque ${ }^{3}$

Descritores

Instituição de longa permanência para idosos Transtornos de deglutição Idoso

Fatores de risco Deglutição Demência

Keywords

Homes for the aged Deglutition disorders Aged Risk factors Deglutition Dementia

Endereço para correspondência: Francelise Pivetta Roque R. José Correia Filho, 282/805, Ponta Verde, Maceió (AL), Brasil, CEP: 57035-130.

E-mail: franceliseroque@yahoo.com.br

Recebido em: 15/7/2011

Aceito em: 15/2/2012

\section{Fatores associados a sinais sugestivos de disfagia orofaríngea em idosas institucionalizadas}

\author{
Factors associated to suggestive signs of oropharyngeal \\ dysphagia in institutionalized elderly women
}

\section{RESUMO}

Objetivo: Identificar os fatores associados a sinais sugestivos de disfagia orofaríngea em idosas institucionalizadas. Métodos: Realizou-se estudo transversal com 30 idosas institucionalizadas (média de idade: $83,73 \pm 10,56$ anos) em Maceió. Coletaram-se, de agosto de 2007 a julho de 2008, dados sociodemográficos, culturais, de saúde geral e de deglutição, mediante análise do prontuário e acompanhamento (presencial e por filmagem) de uma refeição de cada idosa. Compararam-se as idosas com e sem sinais sugestivos de disfagia orofaríngea e foram verificados os fatores associados aos sinais sugestivos de disfagia orofaríngea e às alterações da dinâmica alimentar. Resultados: No grupo de idosas com sinais sugestivos de disfagia verificou-se maior uso de medicação, menor ocorrência de depressão, maior número de dentes e de alterações da dinâmica alimentar. Em relação aos fatores associados às alterações da dinâmica alimentar, as idosas com demência apresentaram mais chances de requererem assistência durante a alimentação e de apresentarem quatro ou mais alterações da dinâmica alimentar. As idosas com assistência requerida apresentaram mais chances de se alimentarem na cama, de estarem posicionadas inadequadamente e de apresentarem três ou mais alterações da dinâmica alimentar. Conclusão: Em idosas institucionalizadas, são considerados fatores associados aos sinais sugestivos de disfagia orofaríngea o uso de medicação, a ausência de depressão, o número de dentes presentes e o número de alterações da dinâmica alimentar apresentadas pelas idosas. Os fatores associados às alterações da dinâmica alimentar estão relacionados à demência e assistência requerida.

\begin{abstract}
Purpose: To identify the factors associated to suggestive signs of oropharyngeal dysphagia (OD) in institutionalized elderly women. Methods: A cross-sectional, study with 30 institutionalized elderly women (mean age: 83,73 10,56 years) was carried out in Maceió. From August 2007 to July 2008, social, demographic and cultural data was collected, as well as data on general health and deglutition from their medical records and follow-up (both in person and by video) of one meal of each elderly woman. Those with signs suggestive of OD were compared to those with no signs of it and the factors associated with signs suggestive of orophayngeal dysphagia and changes in the feeding dynamics were considered. Results: Regarding the factors associated to changes in feeding dynamics, the elderly with dementia were more likely to require assistance during mealtime and to present four or more changes in feeding dynamics. The elderly that required assistance were more likely to eat in bed, at an inadequate position and to have three or more changes in feeding dynamics. Conclusion: For institutionalized elderly women, the factors associated to signs suggestive of oropharyngeal dysphagia were use of medication, absence of depression, number of teeth and number of changes in feeding dynamics. The factors associated to changes in feeding dynamics are related to dementia and need of special care.
\end{abstract}

Trabalho realizado no Curso de Fonoaudiologia, Universidade de Ciências da Saúde de Alagoas - UNCISAL Alagoas (AL), Brasil.

(1) Curso de Fonoaudiologia, Centro de Ciências da Saúde, Universidade Estadual de Ciências da Saúde de Alagoas - UNCISAL - Alagoas (AL), Brasil.

(2) Departamento de Fonoaudiologia, Escola Paulista de Medicina da Universidade Federal de São Paulo UNIFESP - São Paulo (SP), Brasil.

(3) Núcleo de Saúde do Adulto e do Idoso, Centro de Ciências da Saúde, Universidade Estadual de Ciências da Saúde de Alagoas - UNCISAL - Alagoas (AL), Brasil.

Conflito de interesse: nada a declarar. 


\section{INTRODUÇÃO}

A Instituição de Longa Permanência para Idosos (ILPI) constitui uma importante modalidade de atenção ao idoso no Brasil, pois apesar de ser baixo o número de instituições e de idosos nelas residentes (menos de 1\% dos idosos brasileiros ${ }^{(1)}$ ), recebe pessoas consideradas frágeis ou em situação de fragilidade $^{(2)}$. As mulheres idosas, que constituem aproximadamente $57,3 \%$ dos residentes em ILPIs brasileiras, experimentam uma probabilidade maior de ficarem viúvas, em situação socioeconômica desvantajosa e com necessidades especiais ${ }^{(1,2)}$.

Entre os distúrbios de saúde vivenciados por esta população, têm alta frequência a disfagia e as dificuldades encontradas nos momentos da refeição ("mealtime difficulties"), nesse artigo nomeadas como alterações da dinâmica alimentar. Também é alta a ocorrência de broncoaspiração, cujas complicações podem piorar lentamente o estado clínico ${ }^{(3-5)}$, constituindo-se como importante causa de morte em idosos institucionalizados ${ }^{(4)}$.

Estudos internacionais verificaram que a disfagia orofaríngea em residentes de ILPIs está frequentemente relacionada a Acidente Vascular Encefálico (AVE), dependência, demência avançada, higiene oral precária, múltiplos diagnósticos, além de fatores ambientais ${ }^{(6)}$. Os problemas vivenciados pelos idosos durante a refeição não se restringem aos sinais disfágicos incluem alterações cognitivas, comportamentais, físicas e ambientais que, conjuntamente, predispõem o idoso à pneumonia broncoaspirativa, desnutrição e desidratação ${ }^{(7)}$. No que tange à literatura brasileira, foram localizados dois artigos referentes à descrição e caracterização do momento de alimentação de idosas institucionalizadas, em regiões sociodemográfica e culturalmente diferentes, sem a verificação, porém, dos fatores associados à dificuldade de deglutição ${ }^{(8,9)}$.

A identificação dos fatores associados à disfagia orofaríngea em idosos institucionalizados permite o mapeamento da população de risco. Isso possibilita o gerenciamento dos problemas de alimentação e deglutição desta população, a fim de determinar intervenções, individuais e coletivas, tanto fonoaudiológicas como interdisciplinares, visando não somente ao bem estar de cada idoso e seus familiares, quanto à redução de custos em saúde ${ }^{(10)}$.

Desta forma, este estudo teve como objetivo identificar os fatores associados a sinais sugestivos de disfagia orofaríngea em idosas institucionalizadas.

\section{MÉTODOS}

O projeto desta pesquisa foi analisado e aprovado pelo Comitê de Ética em Pesquisa da Universidade Estadual de Ciências da Saúde de Alagoas - UNCISAL, sob o protocolo número 788. Todos os sujeitos envolvidos e/ou seus tutores legais assinaram o Termo de Consentimento Livre e Esclarecido, consentindo a realização e divulgação deste estudo, conforme Resolução 196/96 (BRASIL. Resolução MS/CNS/CNEP $n^{\circ}$ 196/96 de 10 de outubro de 1996).

Trata-se de um estudo transversal analítico. Foram utilizadas informações já coletadas e armazenadas em um banco de dados de pesquisa anterior ${ }^{(9)}$, relativas aos aspectos da dinâmica alimentar e de deglutição de 30 idosas (média de idade:
83,73 $\pm 10,56$ anos) residentes de uma ILPI de Maceió, Alagoas, de caráter filantrópico, exclusiva para mulheres.

O número de idosas incluídas correspondeu ao total de idosas residentes no período em que as filmagens foram realizadas (agosto a dezembro de 2007). Adotou-se como critério de exclusão a utilização de via alternativa de alimentação exclusiva à época do estudo (sonda nasogástrica, nasoenteral, ou ostomia). Porém, nenhuma das residentes se encontrava nessa condição.

A opção pela utilização destes dados, ao invés de se coletar outros mais recentes, deu-se, principalmente, pelo fato de não haver, atualmente, na cidade de Maceió, nenhuma ILPI que seja assistida por uma equipe interdisciplinar especializada em Saúde do Idoso, como era esta à época. Buscou-se eliminar, desta forma, vieses de pesquisa decorrentes de um acompanhamento profissional em saúde insuficiente ou inadequado. Além disto, esses dados não haviam sido analisados sob esta perspectiva, os instrumentos utilizados continuam atuais, e o tempo transcorrido não trouxe mudanças relevantes nos preceitos que embasam o acompanhamento em saúde dos distúrbios de saúde analisados no ambiente institucional.

As idosas recebiam assistência de cuidadoras formais, pagas pela instituição, ou pela própria idosa ou sua família. À época em que os dados foram coletados, as cuidadoras haviam passado por uma capacitação com a duração de 20 horas, que contemplou, entre os temas abordados, os cuidados com a alimentação e a deglutição dos idosos.

Os dados foram coletados mediante análise do prontuário, e acompanhamento (presencial e por meio de filmagem) de uma refeição (almoço) de cada idosa. Foram coletados no prontuário os dados de identificação (idade, estado civil e escolaridade), tempo de institucionalização, diagnósticos médicos, medicações utilizadas (número e categorias), e "status" cognitivo, medido pelo Mini Exame do Estado Mental (MEEM) ${ }^{(11)}$,"status" nutricional, medido pela Mini Avaliação Nutricional (MAN) ${ }^{(12)}$ e grau de independência nas atividades básicas de vida diária, medida pelo índice de Katz ${ }^{(1)}$. Também foram coletados em prontuário os dados da avaliação da deglutição, realizada por uma fonoaudióloga especialista em gerontologia e em motricidade orofacial, com dez anos de experiência no atendimento a idosos. Essa havia sido realizada, como rotina de atendimento da Instituição, há no mínimo um e no máximo dez dias de cada filmagem da refeição. , Os sinais sugestivos de disfagia orofaríngea (DOF) foram registrados com base no Protocolo fonoaudiológico de avaliação do risco para disfagia - PARD ${ }^{(13)}$, utilizando-se, para o presente estudo, os seguintes dados: escape oral anterior, resíduos alimentares após a deglutição, alteração vocal após a deglutição, alteração focal após a deglutição, fase oral aumentada, comportamento de cuspir o alimento ou a saliva, comportamento de morder o utensílio, movimento de língua antagônico à entrada de alimento, tosse durante a alimentação, engasgo, fadiga/alteração respiratória, ausculta cervical alterada.

As filmagens foram realizadas por dois estudantes de graduação em Fonoaudiologia treinados para tal procedimento, e serviram ao registro dos itens "aspectos atitudinais e comportamentais relacionados à alimentação" e "dependência alimentar e assistência requerida/provida no momento da alimentação", cuja análise foi realizada por uma fonoaudióloga, especialista em 
gerontologia e em motricidade orofacial, com dez anos de experiência no atendimento a idosos com alterações de deglutição.

Neste estudo, considerou-se a presença ou ausência de cada um dos sinais indicativos de disfagia orofaríngea, e não o diagnóstico desse distúrbio, primeiramente pelo não acesso, no meio em que houve a pesquisa, a um exame de deglutição complementar à avaliação clínica, que validaria o diagnóstico com maior fidedignidade. O método adotado pressupõe somente a verificação da presença ou ausência de sinais observáveis e, portanto, é menos susceptível a vieses de interpretação. Em se tratando de uma pesquisa que subsidie a triagem dos fatores de risco, a superestimação é preferível à subestimação dos possíveis indicadores de disfagia orofaríngea. Sendo assim, as idosas foram separadas em dois grupos: aquele em que as idosas apresentavam pelo menos um sinal sugestivo de DOF, e aqueles em que as idosas não apresentavam nenhum desses sinais.

Os demais itens, denominados "Alterações da Dinâmica Alimentar" ("Características da refeição/dieta"; "Ambiente de alimentação"; "Aspectos bucais") foram registrados no momento real da alimentação ou próximo a esta, utilizando-se um protocolo desenvolvido a partir dos itens de um instrumento de um estudo norteamericano ${ }^{(7)}$. Os itens, as respostas possíveis e os critérios de análise para cada resposta foram descritos detalhadamente em estudo anterior de mesma autoria desse $\operatorname{artigo}^{(9)}$. Esses registros foram obtidos por um dos dois estudantes responsáveis pela filmagem, treinados para a realização destes registros, tendo sido julgados aptos para tal após a coincidência em cinco registros seguidos de cada item, em outros idosos, não incluídos neste estudo.

Cada refeição foi observada no seu local habitual, utilizando-se, para a filmagem, uma câmera digital Sony ${ }^{\circledR}$
DSC-T10 com resolução máxima de 640x480 pixels e tripé compatível, montados a uma distância que possibilitou a visualização da idosa e, quando pertinente, da sua cuidadora. Ambas foram instruídas a agir naturalmente durante toda a filmagem, cuja duração correspondeu à refeição completa. $\mathrm{O}$ pesquisador responsável pela filmagem esteve presente no início e após o final desta, a fim de que os sujeitos pudessem usufruir a maior parte do tempo a sós, como numa alimentação habitual, visando-se à preservação, o máximo possível, da naturalidade do momento.

Os dados foram apresentados de forma descritiva e analítica. Compararam-se as idosas com e sem sinais sugestivos de disfagia orofaríngea (DOF), utilizando-se o Teste Exato de Fisher para as variáveis categóricas e o Teste de Mann-Whitney para as variáveis escalares. Por meio de análise univariada, estimaram-se os valores de Odds Ratio brutos, com intervalo de confiança de $95 \%$, para pesquisar os fatores associados aos sinais sugestivos de DOF e às alterações da dinâmica alimentar. Para tal, as variáveis foram dicotomizadas. Adotou-se o nível de significância de 5\% $(\mathrm{p}<0,05)$.

\section{RESULTADOS}

A presença de sinais sugestivos de disfagia orofaríngea alcançou uma frequência de ocorrência de 63,3\% nesta amostra. Foram obtidos os dados sociodemográficos e culturais das idosas institucionalizadas segundo a presença ou ausência de sinais sugestivos de DOF (Tabela 1).

Foram analisados, ainda, os dados de saúde das idosas institucionalizadas com DOF (Tabela 2).

Tabela 1. Dados sociodemográficos e culturais das idosas institucionalizadas com e sem sinais sugestivos de disfagia orofaríngea

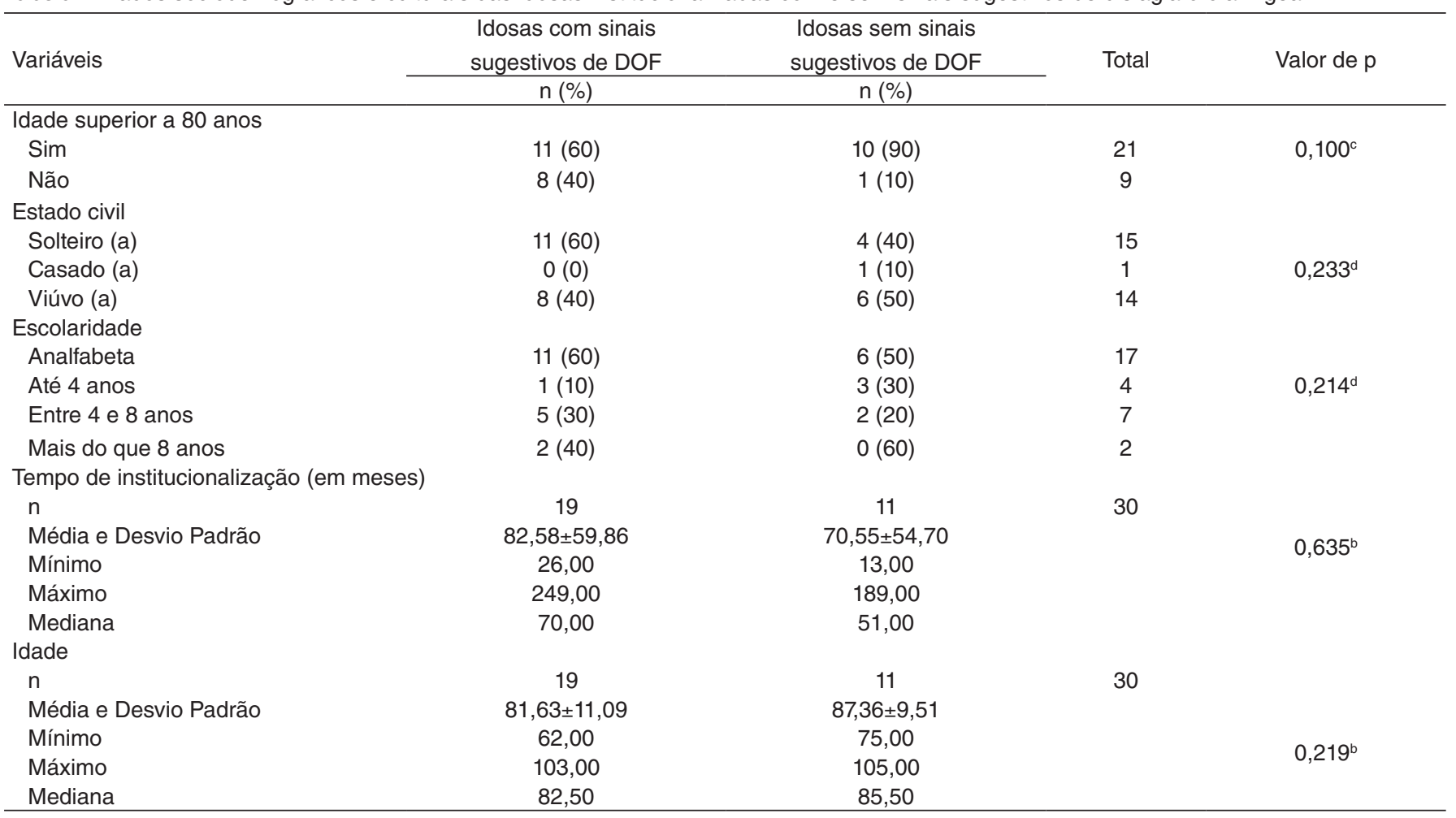

Legenda: DOF = disfagia orofaríngea; ${ }^{\mathrm{b} T e s t e}$ de Mann Whitney; ${ }^{\mathrm{C}}$ Teste Exato de Fisher; ${ }^{\mathrm{d} T e s t e ~ d e ~ R a z a ̃ o ~ d e ~ V e r o s s i m i l h a n c ̧ a ~}$ 
Tabela 2. Dados de saúde das idosas institucionalizadas com e sem sinais sugestivos de disfagia orofaríngea (DOF)

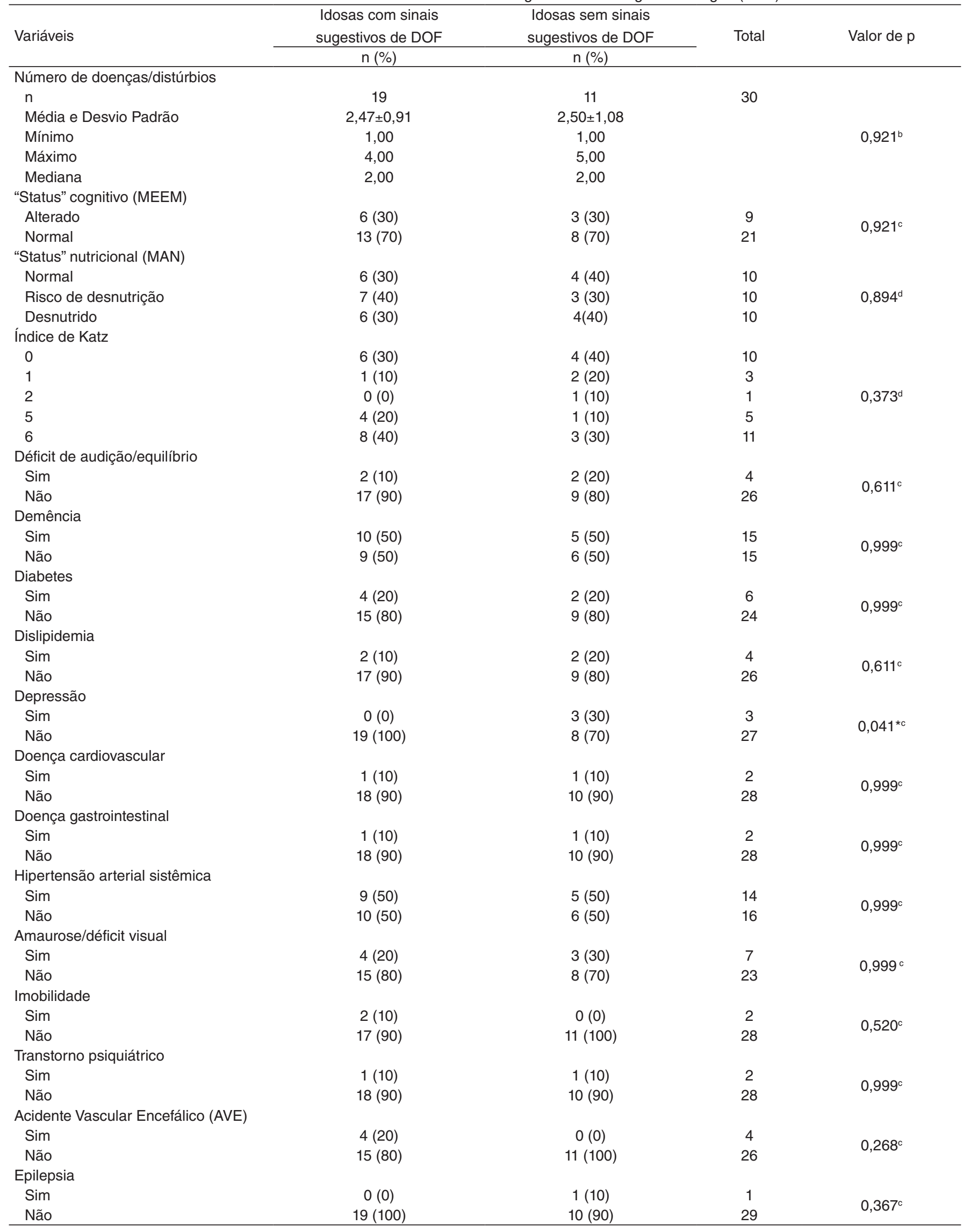


Tabela 2. Continuação

\begin{tabular}{|c|c|c|c|c|}
\hline Variáveis & $\begin{array}{c}\text { Idosas com sinais } \\
\text { sugestivos de DOF } \\
\mathrm{n}(\%)\end{array}$ & $\begin{array}{c}\text { Idosas sem sinais } \\
\text { sugestivos de DOF } \\
\mathrm{n}(\%)\end{array}$ & Total & Valor de $p$ \\
\hline \multicolumn{5}{|l|}{ Osteoartrite } \\
\hline Sim & $2(10)$ & $0(0)$ & 2 & \multirow{2}{*}{$0,520^{\circ}$} \\
\hline Não & $17(90)$ & $11(100)$ & 28 & \\
\hline \multicolumn{5}{|l|}{ Hipotireoidismo } \\
\hline Sim & $2(10)$ & $0(0)$ & 2 & \multirow{2}{*}{$0,520^{\circ}$} \\
\hline Não & $17(90)$ & $11(100)$ & 28 & \\
\hline \multicolumn{5}{|c|}{ Doença Pulmonar Obstrutiva Crônica (DPOC) } \\
\hline Sim & $1(10)$ & $1(0)$ & 2 & \multirow{2}{*}{$0,999^{c}$} \\
\hline Não & $18(90)$ & $10(100)$ & 28 & \\
\hline \multicolumn{5}{|l|}{ Uso de medicação } \\
\hline Sim & $18(90)$ & $7(60)$ & 25 & \multirow{2}{*}{$0,047^{\star c}$} \\
\hline Não & $1(10)$ & $4(40)$ & 5 & \\
\hline \multicolumn{5}{|c|}{ Número de medicamentos em uso } \\
\hline $\mathrm{n}$ & 19 & 11 & 30 & \multirow{5}{*}{$0,122^{b}$} \\
\hline Média e Desvio Padrão & $3,47 \pm 1,65$ & $2,18 \pm 2,23$ & & \\
\hline Mínimo & 0,00 & 0,00 & & \\
\hline Máximo & 6.00 & 6,00 & & \\
\hline Mediana & 3,00 & 1,00 & & \\
\hline \multicolumn{5}{|c|}{ Uso de medicamento anti-hipertensivo } \\
\hline Sim & $11(60)$ & $5(50)$ & 16 & \multirow{2}{*}{$0,707^{c}$} \\
\hline Não & $8(40)$ & $6(50)$ & 14 & \\
\hline \multicolumn{5}{|c|}{ Uso de medicamento antidepressivo } \\
\hline Sim & $2(10)$ & $3(30)$ & 5 & \multirow[b]{2}{*}{$0,327^{c}$} \\
\hline Não & $17(90)$ & $8(70)$ & 25 & \\
\hline \multicolumn{5}{|c|}{ Uso de medicamento anticolinérgico } \\
\hline Sim & $1(10)$ & $0(0)$ & 1 & \multirow{2}{*}{$0,999^{c}$} \\
\hline Não & $18(90)$ & $11(100)$ & 29 & \\
\hline \multicolumn{5}{|c|}{ Uso de medicamento anticonvulsivante } \\
\hline Sim & $2(10)$ & $1(10)$ & 3 & \multirow{2}{*}{$0,999^{c}$} \\
\hline Não & $17(90)$ & $10(90)$ & 27 & \\
\hline \multicolumn{5}{|c|}{ Uso de medicamento anti-histamínico } \\
\hline Sim & $0(0)$ & $0(0)$ & 0 & \multirow{2}{*}{$0,999^{c}$} \\
\hline Não & $19(100)$ & $11(100)$ & 30 & \\
\hline \multicolumn{5}{|l|}{ Uso de benzodiazepínico } \\
\hline Sim & $1(10)$ & $2(20)$ & 3 & \multirow{2}{*}{$0,537^{c}$} \\
\hline Não & $18(90)$ & $9(80)$ & 27 & \\
\hline Uso de neuroléptico & & & & \\
\hline Sim & $3(20)$ & $2(20)$ & 5 & \\
\hline Não & $16(80)$ & $9(80)$ & 25 & $0,999^{c}$ \\
\hline $\begin{array}{l}\text { Uso de medicamentos qu } \\
\text { disfagia orofaríngea }\end{array}$ & & & & \\
\hline Sim & $16(80)$ & $5(50)$ & 21 & \\
\hline Não & $3(20)$ & $6(50)$ & 9 & $0,042^{\times c}$ \\
\hline
\end{tabular}

*Valores significativos $(p<0,05)$

Legenda: DOF = disfagia orofaríngea; ${ }^{\mathrm{b} T e s t e}$ de Mann Whitney; ${ }^{\mathrm{C} T e s t e}$ Exato de Fisher; ${ }^{\mathrm{i}}$ Teste de Razão de Verossimilhança

Quanto aos aspectos bucais e os dados da dinâmica alimentar das idosas institucionalizadas, foram comparadas as idosas com e sem sinais sugestivos de DOF (Tabela 3).

Foram visualizados os fatores que se mostraram associados, na análise de risco univariada, às alterações da dinâmica alimentar. Nesta análise, não houve associação aos sinais sugestivos de DOF (Tabela 4).

Utilizando-se o programa GPower 3.0, calcularam-se o tamanho da amostra e o poder do estudo. Para o primeiro, utilizou-se a variável "número de dentes", em função da presença/ ausência de disfagia, adotando-se o alfa (erro tipo I) de 0,050 $(5 \%)$, poder $(1-\beta)$ de $0,800(80 \%)$, razão entre os tamanhos dos grupos estudados igual a 1, chegando-se ao tamanho da amostra de 32 sujeitos. Para o cálculo do poder do estudo, adotou-se alfa (erro tipo I) de 0,050 (5\%), tamanho do grupo 1 de 19 sujeitos, tamanho do grupo 2 de 11 sujeitos, razão entre os tamanhos dos grupos estudados igual a 1, chegando-se ao poder calculado de $0,751(75,1 \%)$.

\section{DISCUSSÃO}

A maioria dos estudos realizados em Instituições de Longa Permanência para Idosos (ILPI) avaliam os fatores associados à pneumonia aspirativa ${ }^{(4,14)}$, à desnutriçãa ${ }^{(15)} \mathrm{e}$ à dependência para 
Tabela 3. Dados bucais e da dinâmica alimentar das idosas institucionalizadas com e sem sinais sugestivos de disfagia orofaríngea

\begin{tabular}{|c|c|c|c|c|}
\hline \multirow[b]{2}{*}{ Variáveis } & Idosas com Sinais & Idosas sem Sinais & \multirow[b]{2}{*}{ Total } & \multirow[b]{2}{*}{ Valor de $p$} \\
\hline & Sugestivos de DOF & Sugestivos de DOF & & \\
\hline \multicolumn{5}{|l|}{ Número de dentes } \\
\hline Média e Desvio Padrão & $7,26 \pm 7,64$ & $2,00 \pm 3,10$ & & \\
\hline Mínimo & 0,00 & 0,00 & & \\
\hline Máximo & 28,00 & 8,00 & & \\
\hline Adequado & $12(63)$ & $4(36)$ & 16 & $0,999^{c}$ \\
\hline Inadequado & $1(5)$ & $0(0)$ & 1 & \\
\hline Não se aplica (ausência de dentes) & $6(32)$ & $7(64)$ & 13 & \\
\hline \multicolumn{5}{|l|}{ Higiene oral } \\
\hline Inadequada & $18(90)$ & $11(100)$ & 29 & $0,999^{c}$ \\
\hline \multicolumn{5}{|l|}{ Uso de prótese dentária total superior } \\
\hline Ausente & $14(70)$ & $7(60)$ & 21 & $0,867^{c}$ \\
\hline Presente & $5(30)$ & $4(40)$ & 9 & \\
\hline \multicolumn{5}{|c|}{ Tempo total de alimentação (em minutos) } \\
\hline $\mathrm{n}$ & 19 & 11 & 30 & $0,589^{b}$ \\
\hline Média e Desvio Padrão & $10,58 \pm 6,16$ & $8,82 \pm 4,49$ & & \\
\hline Mínimo & 3,00 & 3,00 & & \\
\hline Máximo & 21,00 & 17,00 & & \\
\hline Mediana & 10,00 & 9,00 & & \\
\hline Tempo total de alimentação & & & & $0,499^{d}$ \\
\hline 0 a $5 \min$ & $4(20)$ & $3(30)$ & 7 & \\
\hline 6 a $10 \mathrm{~min}$ & $8(40)$ & $4(40)$ & 12 & \\
\hline \multicolumn{5}{|c|}{ Adequação da quantidade de alimento ingerido } \\
\hline Sim & $14(70)$ & $8(70)$ & 22 & $0,999^{c}$ \\
\hline Não & $5(30)$ & $3(30)$ & 8 & \\
\hline \multicolumn{5}{|l|}{ Ambiente - Iluminação } \\
\hline Claro & $16(80)$ & $9(80)$ & 25 & $0,999^{c}$ \\
\hline Escuro & $3(20)$ & $2(20)$ & 5 & \\
\hline \multicolumn{5}{|l|}{ Ambiente acústico } \\
\hline Silencioso & $8(40)$ & $6(50)$ & 14 & $0,707^{c}$ \\
\hline Ruidoso & $11(60)$ & $5(50)$ & 16 & \\
\hline \multicolumn{5}{|l|}{ Presença de distratores visuais } \\
\hline Sim & $10(50)$ & $3(30)$ & 13 & $0,259^{c}$ \\
\hline Não & $9(50)$ & $8(70)$ & 17 & \\
\hline \multicolumn{5}{|c|}{ Posicionamento das idosas (se no quarto) } \\
\hline $60^{\circ}$ & $3(20)$ & $0(0)$ & 3 & $0,279^{c}$ \\
\hline $90^{\circ}$ & $16(80)$ & $11(100)$ & 27 & \\
\hline \multicolumn{5}{|l|}{ Adequação do posicionamento } \\
\hline Inadequado & $9(50)$ & $2(20)$ & 11 & $0,140^{c}$ \\
\hline Adequado & $10(50)$ & $9(80)$ & 19 & \\
\hline Atitude em relação à alimentação & & & & \\
\hline Colaborativa & $16(80)$ & $10(90)$ & 26 & $0,999^{c}$ \\
\hline Não Colaborativa & $3(20)$ & $1(10)$ & 4 & \\
\hline Problema de Comportamento & & & & \\
\hline Sim & $12(60)$ & $4(40)$ & 16 & $0,257^{c}$ \\
\hline Não & $7(40)$ & $7(60)$ & 14 & \\
\hline
\end{tabular}


Tabela 3. Continuação

\begin{tabular}{|c|c|c|c|c|}
\hline \multirow{3}{*}{ Variáveis } & Idosas com Sinais & Idosas sem Sinais & \multirow{3}{*}{ Total } & \multirow{3}{*}{ Valor de $p$} \\
\hline & Sugestivos de DOF & Sugestivos de DOF & & \\
\hline & $\mathrm{n}(\%)$ & $\mathrm{n}(\%)$ & & \\
\hline \multicolumn{5}{|l|}{ Assistência requerida } \\
\hline Sim & $13(70)$ & $4(40)$ & 17 & \multirow[t]{2}{*}{$0,132^{c}$} \\
\hline Não & $6(30)$ & $7(60)$ & 13 & \\
\hline \multicolumn{5}{|l|}{ Assistência provida } \\
\hline Sim & $8(40)$ & $3(30)$ & 11 & \multirow[t]{2}{*}{$0,466^{c}$} \\
\hline Não & $11(60)$ & $8(70)$ & 19 & \\
\hline \multicolumn{5}{|l|}{ Cospe o alimento ou saliva } \\
\hline Sim & $4(20)$ & $0(0)$ & 4 & \multirow[t]{2}{*}{$0,268^{c}$} \\
\hline Não & $15(80)$ & $11(100)$ & 26 & \\
\hline \multicolumn{5}{|c|}{ Inadequação da quantidade de alimento ingerida por vez } \\
\hline Sim & $7(40)$ & $4(40)$ & 11 & \multirow[t]{2}{*}{$0,999^{c}$} \\
\hline Não & $12(60)$ & $7(60)$ & 19 & \\
\hline \multicolumn{5}{|c|}{ Inadequação da velocidade de ingestão } \\
\hline Sim & $5(30)$ & $6(50)$ & 11 & \multirow[t]{2}{*}{$0,238^{c}$} \\
\hline Não & $14(70)$ & $5(50)$ & 19 & \\
\hline \multicolumn{5}{|c|}{ Presença de alterações da dinâmica alimentar } \\
\hline Sim & $19(100)$ & $11(100)$ & 30 & \multirow[t]{2}{*}{$0,999^{c}$} \\
\hline Não & $0(0)$ & $0(0)$ & & \\
\hline \multicolumn{5}{|c|}{ Número de pessoas no local } \\
\hline $\mathrm{n}$ & 19 & 11 & & \multirow[t]{5}{*}{$0,681^{b}$} \\
\hline Média e Desvio Padrão & $7,32 \pm 7,01$ & $6,64 \pm 8,29$ & & \\
\hline Mínimo & 0,00 & 0,00 & & \\
\hline Máximo & 20,00 & 25,00 & & \\
\hline Mediana & 4,00 & 2.50 & & \\
\hline \multicolumn{5}{|c|}{ Número de alterações da dinâmica alimentar } \\
\hline $\mathrm{n}$ & 19 & 11 & & \multirow[t]{5}{*}{$0,016^{* b}$} \\
\hline Média e Desvio Padrão & $4,42 \pm 1,77$ & $2,91 \pm 1,92$ & & \\
\hline Mínimo & 1,00 & 1,00 & & \\
\hline Máximo & 8,00 & 8,00 & & \\
\hline Mediana & 5,00 & 2,00 & & \\
\hline
\end{tabular}

${ }^{*}$ Valores significativos $(p<0,05)$

Legenda: DOF = disfagia orofaríngea; ${ }^{\mathrm{b} T e s t e}$ de Mann Whitney; ${ }^{\mathrm{c} T e s t e}$ Exato de Fisher; ${ }^{\mathrm{d} T e s t e}$ de Razão de Verossimilhança

Tabela 4. Fatores associados às alterações da dinâmica alimentar

\begin{tabular}{|c|c|c|c|c|}
\hline \multicolumn{3}{|c|}{ Dependência para alimentação } & \multirow{3}{*}{ OR (IC95\%) } & \multirow{3}{*}{ Valor de $\mathrm{p}$} \\
\hline & \multicolumn{2}{|c|}{ Assistência requerida } & & \\
\hline Diagnóstico de demência & Sim & Não & & \\
\hline Sim & 13 & 2 & \multirow[b]{2}{*}{$17,87(2,7-116,8)$} & \multirow[b]{2}{*}{$0,003^{*}$} \\
\hline Não & 4 & 11 & & \\
\hline Local específico de alimentação - cama & \multicolumn{4}{|c|}{ Ambiente para alimentação } \\
\hline Assistência requerida & Sim & Não & \multirow{3}{*}{$7,85(1,3-47,04)$} & \multirow{3}{*}{$0,04^{*}$} \\
\hline Sim & 10 & 7 & & \\
\hline Não & 2 & 11 & & \\
\hline \multicolumn{5}{|c|}{ Inadequação do posicionamento } \\
\hline Assistência requerida & Sim & Não & \multirow{3}{*}{$17,14(1,7-163,81)$} & \multirow{3}{*}{$0,01^{*}$} \\
\hline Sim & 10 & 7 & & \\
\hline Não & 1 & 12 & & \\
\hline \multicolumn{5}{|c|}{$\begin{array}{c}\text { Alterações da dinâmica alimentar } \\
\text { Número de alterações }\end{array}$} \\
\hline Diagnóstico de demência & $4+$ & $0-3$ & \multirow{3}{*}{$11,00(1,9-60,5)$} & \multirow{3}{*}{$0,01^{*}$} \\
\hline Sim & 12 & 3 & & \\
\hline Não & 4 & 11 & & \\
\hline \multicolumn{5}{|c|}{ Número de alterações } \\
\hline Assistência requerida & $3+$ & $0-2$ & \multirow{3}{*}{$36,00(3,4-373,1)$} & \multirow{3}{*}{$0,001^{*}$} \\
\hline Sim & 16 & 1 & & \\
\hline Não & 4 & 9 & & \\
\hline
\end{tabular}

*Valores significativos $(p<0,05)$

Legenda: $\mathrm{OR}=$ Odds Ratio 
alimentação ${ }^{(16)}$, não à disfagia orofaríngea, nem aos seus sinais sugestivos. Quando objetivam analisar a deglutição, fazem-no de forma descritiva, incluindo-se, por vezes, outros aspectos da dinâmica alimentar, ou apresentando a frequência da disfagia $^{(3,5,5,9)}$. Isso limita a discussão dos dados desta pesquisa com os demais trabalhos existentes na literatura.

A presença de sinais sugestivos de DOF alcançou uma frequência de $63,3 \%$ nesta população, e, portanto, se encontra de acordo com a variação encontrada na literatura $(26,5 \text { a } 87 \%)^{(5,7)}$. Apesar de a amostra, exclusivamente composta por mulheres, não ter permitido a testagem da associação entre a variável gênero e a provável disfagia, em estudo anterior, tal variável não se mostrou fator de risco para a presença de pneumonia broncoaspirativa ${ }^{(4)}$.

A não-associação entre os dados sociodemográficos e culturais e os sinais sugestivos de DOF contrariou os achados de que tanto a idade, quanto o fato de ser muito idoso (ter 80 anos ou mais nos países em desenvolvimento, e 85 anos ou mais nos países desenvolvidos), são fatores de risco para a pneumonia broncosaspirativa ${ }^{(4)}$. Em geral, o perfil de "muito idosos" (80 anos ou mais) é constituído por indivíduos com demência ${ }^{(5)}$, várias comorbidades e dependência, que comprometem diversas atividades da vida diária (AVDs), incluindo-se a alimentação ${ }^{(16)}$. Eles compõem, assim, um grupo frágil que requer maior assistência, em consonância com os dados apresentados neste estudo.

A ausência de associação entre a escolaridade e a presença dos sinais sugestivos de DOF concorda com dados obtidos em estudo anterior, que também não verificou a influência da escolaridade na pneumonia broncoaspirativa ao comparar idosos com e sem dentes ${ }^{(17)}$. O estado civil e o tempo de institucionalização não foram analisados, nos estudos revisados, como fatores de risco para disfagia ou aspectos possivelmente a elas associados, como pneumonia ou desnutrição. Sabe-se, porém da influência desses fatores no estado de saúde dessa população ${ }^{(18)}$, e que poderiam, portanto, demonstrar a associação com a provável disfagia.

Quanto à análise dos fatores de saúde, a ausência de associação entre os sinais sugestivos de DOF e o número de diagnósticos médicos contrariou nossas expectativas, visto que a literatura os aponta como fator importante para pneumonia broncoaspirativa ${ }^{(6)}$. A prevalência de comorbidades na velhice tende a ser alta conforme a idade avança ${ }^{(19)}$. Residentes de ILPIs têm condições médicas crônicas que frequentemente e gradualmente conduzem à "descompensação" do estado funcional, estado nutricional e pulmonar ${ }^{(3)}$.

A ausência de associação entre demência ou alteração cognitiva e os sinais sugestivos de DOF contrariou os achados de estudos anteriores ${ }^{(5,6)}$.

A não associação entre alteração do estado nutricional e os sinais sugestivos de DOF encontrada nesse estudo contrariou os resultados de estudo que, analisando todas as ILPIs de Helsinki, na Finlândia, encontrou as dificuldades de deglutição como fator preditivo para a desnutrição ${ }^{(15)}$. Uma hipótese explicativa para os dados da presente pesquisa é a de que essas idosas estavam em acompanhamento multiprofissional, com geriatra, nutricionista, fonoaudiólogo e fisioterapeuta, responsáveis por seu gerenciamento nutricional ${ }^{(20)}$.
A ausência de associação entre o nível de dependência nas AVDs, dependência para alimentação (assistência requerida), e sinais sugestivos de DOF, vai de encontro aos dados de estudo que analisou as características e as inabilidades presentes em cada nível de dependência para as atividades de vida diária. Os autores observaram um aumento dos distúrbios de deglutição conforme maior a dependência e pior o status de saúde ${ }^{(21)}$. Também contrariou as evidências científicas de que a dependência para a alimentação é fator de risco para pneumonia decorrente de broncoaspiração ${ }^{(4)}$.

O Diabetes Mellitus foi considerado, em estudo anterior, como sendo fator de risco para pneumonia aspirativa, sob a explicação de predispor o organismo a infecções em geral e à presença de bactérias relacionadas à pneumonia ${ }^{(17)}$. No presente estudo, a doença não se mostrou associada aos sinais sugestivos de DOF.

A associação encontrada entre a ausência de depressão e os sinais sugestivos de DOF contradizem os achados da literatura ${ }^{(22)}$. Porém, isso pode ser explicado com base na dificuldade existente de se diagnosticar esse quadro em idosos com prejuízo cognitivo ${ }^{(23)}$, a exemplo de grande parte da amostra estudada.

A presença de disfagia orofaríngea em doenças cardiológi$\operatorname{cas}^{(24)}$ e gastroenterológicas ${ }^{(25)}$ não foi evidenciada no presente estudo. A associação entre amaurose/déficit visual e as alterações da dinâmica alimentar era esperada, especialmente quando associada a outras deficiências físicas ou cognitivas, tendo-se em vista a relevância da visão para o ato de se alimentar ${ }^{(26)}$. Há que se considerar, porém, que havia tendência de essas idosas terem sido alimentadas por uma cuidadora, o que minimizaria as dificuldades durante a refeição.

Mesmo conhecendo a associação entre os transtornos psiquiátricos e as alterações da dinâmica alimentar que podem influir também na deglutição ${ }^{(27)}$, ela não foi verificada na presente amostra. Ressalta-se que havia somente duas idosas com esse diagnóstico, distribuídas igualmente nos grupos com e sem sinais disfágicos.

O presente estudo não encontrou associação entre Acidente Vascular Encefálico e a presença dos indícios de disfagia, corroborando os resultados de um dos estudos revisados ${ }^{(17)}$. Entretanto, esse achado contraria os resultados da maioria das pesquisas, nas quais se evidencia que tal relação é forte, e que aproximadamente 43 a 53\% dos pacientes com AVE apresentam disfagia, do quais cerca de $37 \%$ desenvolverão pneumonia broncoaspirativa $^{(4,6)}$.

Tendo-se em vista a possibilidade de a epilepsia estar associada a lesões encefálicas ${ }^{(28)}$, a sua relação com a disfagia seria esperada. Porém, seria necessária a presença de mais do que uma idosa na amostra para que tal hipótese pudesse ser testada. A Doença Pulmonar Obstrutiva (DPOC), que nesse estudo não se mostrou associada à provável disfagia orofaríngea, foi verificada como fator de risco para pneumonia aspirativa na literatura $^{(4,17)}$.

Embora os estudos revisados não tenham se proposto a analisar a associação entre as demais doenças/distúrbios de saúde ainda não mencionados nessa discussão, eles foram incluídos na análise por termos objetivado investigar todos os 
dados de saúde disponíveis acerca dessa amostra. A ausência de associação entre esses fatores e os sinais sugestivos de DOF corrobora, de forma indireta, a ausência dessa análise na literatura revisada.

Nesse estudo, a associação entre medicação e sinais sugestivos de DOF ocorreu tanto em relação ao fato de usar medicamentos, independente da classe de medicação, incluindo-se, mas não se restringindo, as que comumente causam disfagia. Os estudos mostram que, em geral, o número de medicamentos utilizados é fator de risco para pneumonia aspirativa em idosos institucionalizados ${ }^{(4,5)}$. Sabe-se ainda que entre as causas de disfagia, a de origem medicamentosa tem grande relevância clínica na população idosa, visto que este grupo de pessoas apresenta maior predisposição ao surgimento dos efeitos indesejáveis devido a maior sensibilidade a essas substâncias. As mais comumente envolvidas no aparecimento do processo são os antipsicóticos, antidepressivos, antiparkinsonianos e anticolinérgicos ${ }^{(25)}$. A associação com provável disfagia ocorreu mediante ao uso de qualquer medicação, mesmo daquelas que não interferem no processo de deglutição. Isso nos remetendo à hipótese explicativa de que este grupo, por apresentar múltiplos diagnósticos, é caracterizado como uma população frágil e, portanto, mais propensa ao aparecimento da disfagia orofaríngea ${ }^{(4,6)}$.

A associação entre o número de dentes e os sinais sugestivos de DOF difere dos resultados de estudo anterior, cujo objetivo era investigar a importância dos fatores médicos e dentais relacionados a pneumonia aspirativa em idosos ${ }^{(17)}$. Embora o presente estudo não tenha evidenciado associação entre o estado de conservação dentária e a higiene oral, a literatura evidencia que a higiene oral inadequada possibilita a proliferação de bactérias Gram negativas anaeróbias, $S$. aureus e de algumas espécies que são potencialmente patógenas do sistema respiratório, o que a torna um fator predisponente ao desenvolvimento de pneumonia broncoaspirativa ${ }^{(6,14,17,22)}$.

Não houve associação entre os sinais de disfagia e as variáveis referentes ao posicionamento da idosa, à quantidade do alimento ingerida por vez, e à velocidade de ingestão. No entanto, sabe-se que a realização das refeições em um posicionamento adequado, com ângulo de $90^{\circ}$ ajuda a prevenir aspiração, e que a forma como o idoso dependente é alimentado pelo cuidador influencia na aspiração de alimento $^{(29)}$.

Embora tenhamos considerado, nesse estudo, como alterações da dinâmica alimentar fatores que são comuns na alimentação de qualquer indivíduo, a exemplo do ruído no ambiente, o número dessas alterações se mostrou fator associado, nesse estudo, à presença dos sinais sugestivos de disfagia orofaríngea. Isso reforça que os problemas alimentares encontrados nas ILPIs não se limitam aos sinais disfágicos. A literatura cita, como alterações na dinâmica alimentar (mealtime difficulties), fatores que, no presente estudo, por vezes não se mostraram associados aos sinais de DOF, tais como a mudança na consistência alimentar ${ }^{(14)}$. Ressalta-se que, nesse estudo, isso pode estar relacionado ao fato de que, apesar das orientações fonoaudiológicas e nutricionais, a ILPI se mostrava resistente ao preparo de consistências adequadas a cada idosa, havendo, portanto, uma tendência à oferta da consistência pastosa mesmo para as idosas que tinham condições de deglutir alimentos semissólidos.

A análise de risco univariada não demonstrou fatores associados aos sinais sugestivos de DOF. Já quanto aos fatores associados às alterações da dinâmica alimentar, observamos associação forte entre a demência e a dependência alimentar, em concordância com a literatura ${ }^{(16,27)}$. Conforme progride a demência, surgem várias inabilidades associadas às alterações cognitivas e comportamentais, que geram impacto na dinâmica alimentar. Tais inabilidades podem ser responsáveis por alterações da deglutição desde a sua fase inicial, em princípio comprometendo os aspectos da fase pré-oral e oral, tornando este grupo candidato ao recebimento de assistência ${ }^{(27)}$.

Ainda no tocante aos fatores associados às alterações da dinâmica alimentar, esse estudo encontrou associação entre as variáveis referentes à necessidade de assistência e alimentação na cama, assim como entre assistência requerida e posicionamento inadequado. A maior dependência da idosa para se alimentar geralmente se associa à maior dependência geral. Isso requer preparo dos cuidadores e da instituição, a exemplo de tecnologias assistivas, para lidar com essas dificuldades de forma que a idosa mantenha o posicionamento adequado e tenha condições de ser transportada para o ambiente adequado de alimentação ${ }^{(16,21)}$.

No que concerne ao número de alterações da dinâmica alimentar, este estudo apontou que há maior requerimento de assistência por pacientes que apresentam três ou mais alterações da dinâmica alimentar. Um estudo verificou a mesma correlação, porém sendo a presença de uma ou mais alterações da dinâmica alimentar fator preditivo para assistência requerida ${ }^{(30)}$.

A associação entre as alterações na dinâmica alimentar e demência e assistência requerida aponta para a relação indireta desses dois últimos com os sinais sugestivos de DOF. Conforme discutido anteriormente, tais sinais se mostraram associados ao número de alterações da dinâmica alimentar.

Esse estudo buscou contribuir no sentido de fornecer informações que embasem ações dos profissionais de saúde, incluindo-se os fonoaudiólogos, a fim de identificarem e gerenciarem os fatores de risco para a disfagia orofaríngea em idosos institucionalizados. Espera-se facilitar a implantação de protocolos de triagem e manejo da deglutição desse público, que tem características peculiares em relação aos idosos residentes em comunidades e hospitalizados.

Tendo-se em vista que o cálculo do tamanho da amostra foi de 32 sujeitos, um número maior de sujeitos poderia conferir maior confiabilidade, desde que houvesse mais sujeitos disponíveis para serem incluídos nessa pesquisa. O poder da amostra, abaixo do ideal, limita, porém não inviabiliza a generalização dos dados.

\section{CONCLUSÃO}

Os fatores associados aos sinais sugestivos de disfagia orofaríngea em idosas institucionalizadas são o uso de medicação, a ausência de depressão, o número de dentes presentes e o número de alterações da dinâmica alimentar apresentadas pelas idosas. A demência associa-se à presença de quatro ou 
mais alterações da dinâmica alimentar e à assistência requerida. Esta última, por sua vez, associa-se à alimentação na cama, à inadequação do posicionamento, e à presença de três ou mais alterações na dinâmica alimentar.

\begin{abstract}
* FMSB participou do delineamento do trabalho, da análise e interpretação dos dados, da redação do artigo e da aprovação da versão a ser publicada; BMC participou da concepção do trabalho, da interpretação dos dados, da revisão crítica do manuscrito e da aprovação da versão a ser publicada; FPR participou da concepção e delineamento do estudo, da análise e interpretação dos dados, da redação do artigo, da sua revisão crítica, e da aprovação da versão a ser publicada.
\end{abstract}

\section{REFERÊNCIAS}

1. Ipea - Instituto de Pesquisa Economia Aplicada. Série Eixos do Desenvolvimento Brasileiro $n^{\circ}$ 93: Infraestrutura Social e Urbana no Brasil subsídios para uma agenda de pesquisa e formulação de políticas públicas. Condições de funcionamento e infraestrutura das instituições de longa permanência para idosos no Brasil. Brasília; 2011. [Internet]. [citado 2011 Maio 29]. Disponível em: http://www.ipea.gov.br/portal/ images/stories/PDFs/comunicado/110524_comunicadoipea93.pdf

2. Brasil. Ministério da Saúde. Portaria n. 2.528, de 19 de outubro de 2006. Aprova a Política Nacional de Saúde da Pessoa Idosa. Diário Oficial da República Federativa do Brasil. Brasília (DF), 20 out. 2006. Seção 1, p. 142.

3. Botella Trelis JJ, Ferrero López MI. Management of dysphagia in the institutionalized elderly patient: current situation. Nutr Hosp. 2002;17(3):168-74.

4. Langmore SE, Skarupski KA, Park PS, Fries BE. Predictors of aspiration pneumonia in nursing home residents. Dysphagia. 2002;17(4):298-307.

5. Irles Rocamora JA, Sánchez-Duque MJ, de Valle Galindo PB, Bernal Lopez E, Fernández Palacín A, Almeida González C, et al. A prevalence study of dysphagia and intervention with dietary counselling in nursing home from Seville. Nutr Hosp. 2009;24(4):498-503.

6. Sue Eisenstadt E. Dysphagia and aspiration pneumonia in older adults. J Am Acad Nurse Pract. 2010;22(1):17-22.

7. Steele CM, Greenwood C, Ens I, Robertson C, Seidman-Carlson R. Mealtime difficulties in a home for the aged: not just dysphagia. Dysphagia. 1997;12(1):43-50; discussion 51.

8. Dias, BKP, Cardoso, MCAF. Caracterísiticas da função de deglutição em um grupo de idosas institucionalizadas. Estud Interdiscipl Envelhec. 2009;14(1):107-24.

9. Roque FP, Bomfim FMS, Chiari BM. Descrição da dinâmica de alimentação de idosas institucionalizadas. Rev Soc Bras Fonoaudiol. 2010;15(2):256-63.

10. Shanley C, O’Loughlin, G. Dysphagia among nursing home residents: an assessment and management protocol. J Gerontol Nurs. 2000; 26(8):35-48.

11. Nitrini R, Caramelli P, Bottino CMC, Damasceno BP, Brucki SMD, Anghinah R. Diagnóstico de Doença de Alzheimer no Brasil: avaliação cognitiva e funcional. Recomendações do Departamento Científico de Neurologia Cognitiva e do Envelhecimento da Academia Brasileira de Neurologia. Arq Neuropsiquiatr 2005;63(3-A):720-7.

12. Raslan M, Gonzalez MC, Dias MCG, Paes-Barbosa FC, Cecconello I, Waitzberg DL. Aplicabilidade dos métodos de triagem nutricional no paciente hospitalizado. Rev Nutr. 2008;21(5): 553-561.

13. Padovani AR, Moraes DP, Mangili LD, Andrade CRF. Protocolo fonoaudiológico de avaliação do risco para disfagia (PARD). Rev Soc Bras Fonoaudiol. 2007;12(3):199-205...

14. Quagliarello V, Ginter S, Han L, Van Ness P, Allore H, Tinetti M. Modifiable risk factors for nursing home-acquired pneumonia. Clin Infect Dis. 2005;40(1):1-6.

15. Suominen M, Muurinen S, Routasalo P, Soini H, Suur-Uski I, Peiponen A, et al. Malnutrition and associated factors among aged residents in all nursing homes in Helsink. Eur J Clin Nutr. 2005Apr; 59(4):578-83.

16. Simmons, SF, Schnelle JF. Feeding assistance needs of long-stay nursing home residents and staff time to provide care. J Am Geriatric Soc. 2006;54 (6):919-24.

17. Terpenning MS, Taylor GW, Lopatin DE, Kerr CK, Dominguez BL, Loesche WJ. Aspiration pneumonia: dental and oral risk factors in an older veteran population. J Am Geriatr Soc. 2001;49(5):557-63.

18. Ramos LR. Fatores determinantes do envelhecimento saudável em idosos residentes em centro urbano: Projeto Epidoso. Cad. Saúde Pública. 2003;19(3):793-7.

19. Pollo SHL, Assis M. Instituições de longa permanência para idosos ILPIS: desafios e alternativas no município do Rio de Janeiro. Rev. Bras. Geriatr. Gerontol. 2008;11(1):29-44.

20. Kikutani T, Enomoto R, Tamura F, Oyaizu K, Suzuki A, Inaba S. Effects of oral functional training for nutritional improvement in Japanese older people requiring long-term care. Gerodontology. 2006;23(2):93-8

21. Imahashi K, Kawagoe M, Eto F, Haga N. Clinical status and dependency of the elderly requiring long-term care in Japan. Tohoku J Exp Med. 2007;212(3):229-38.

22. Yonehama T, Yoshida M, Ohrui T, Mukaiyama H, Okamoto $H$ Hoshiba K, et al. Oral care reduces pneumonia in older patients in nursing homes. J Am Geriatr Soc. 2002Mar;(50):430-3

23. Bazzarella MCS. Critério diagnóstico provisório para depressão na doença de Alzheimer. Rev Psiquiatr Clín. 2003;30(3):102-3.

24. Cheng EM, Fung $\mathrm{CH}$. Quality indicators for the care of stroke and atrial fibrillation in vulnerable elders. J Am Geriatr Soc. 2007;55(2):431-7.

25. Leopold NA, Kagel MC. Dysphagia--ingestion or deglutition?: a proposed paradigm. Dysphagia.1997;12(4):202-6.

26. Sanches EP, Bilton T, Suzuki H, Ramos LR. Estudo da alimentação e deglutição de idosos com doença de Alzheimer leve e moderada. Distúrb. comum. 2003;15(1)9-37.

27. Trentin AP, Teive HAG, Tsubouchi MH, Paola L, Minguetti G. CT findings in 1000 consecutive patients with seizures. Arq. Neuropsiquiatr. 2002;60(2B):416-9.

28. Taylor, IC. Drogas, disfagia e nutrição. Pro Fono. 1997;9(1):41-57.

29. Marik PE, Kaplan D. Aspiration pneumonia and dysphagia in the elderly. Chest. 2003;124(1):328-36.

30. Berkhout AM, Cools HJ, van Houwelingen HC. The relationship between difficulties in feeding oneself and loss of weight in nursing-home patients with dementia. Age Ageing. 1998;27(5):637-41. 